\title{
Manifestaciones de violencia en la escuela primaria: elementos de perfilación de víctimas y agresores
}

\author{
FELIPE ALFONSO ROJAS MONCRIFF \\ CESAR AUGUSTO SIERRA VARÓN
}

Fecha de recepción: 26-06-2009 - Aprobación: 23-09-2009

\section{INTRODUCCIÓN}

Este trabajo presenta una visión general en forma descriptiva de la violencia escolar, basada en una observación naturalista de corte etnográfico, llevada a cabo en una escuela de Bogotá, con niños y niñas que cursan tercer grado de primaria. El propósito de la investigación es la obtención de perfiles de víctimas y agresores, que se encuentran en la relación entre pares escolares.

\section{Resumen}

El objetivo de esta investigación etnográfica fue describir y extraer características de las niñas y los niños agresores y víctimas en el fenómeno de la violencia escolar. Se trabajó con niños y niñas de tercero de primaria de una institución educativa en Bogotá de estrato socioeconómico 2. La metodología fue la observación naturalista no participante, y como instrumentos se utilizaron los diarios de campo y la entrevista semiestructurada, validados por juicios de expertos, cuyo objetivo fue recolectar la información relevante para la investigación. Con base en los datos recogidos gracias a los instrumentos, se detectaron las características particulares de los niños agresores y de los niños víctimas seleccionados. Los resultados se analizaron por las categorías guías del estudio. Así mismo, permitieron considerar las prácticas que se dan en el fenómeno de la violencia escolar. Se encontró que los niños y las niñas, agresores y víctimas, presentan características particulares que permiten una posterior identificación.

\section{Palabras clave}

Violencia escolar, victimización, perfiles.
Las categorías de análisis se formulan y presentan con fundamentación bibliográfica, así como también de acuerdo con las observaciones realizadas. Se contribuye de esta manera con el análisis del fenómeno de la victimización entre escolares. En esta ocasión se hace referencia al fenómeno denominado "bullying", específicamente como lo plantean Olweus (1998) y Ortega

\section{Abstract}

The purpose of this ethnographic research was to describe and to extract characteristics of the bully and victim children within the school violence phenomenon. This research was made with children of third grade of an elementary school in Bogotá of second stratum. The methodology was naturalist non-participant observation, and the instruments were field journals and a semi-structured interview, validated by experts and used to gather relevant data according to the objectives. Based on the information gathered with those instruments, the particular characteristics of the bullies and the victims were detected. The results were analyzed by the categories of the research. Also, they allowed taking into account the practices that take part in the phenomenon of school violence. It was found that bullies and victims present individual characteristics that allow a subsequent identification due to such characteristics.

\section{Key words}

School violence, bullying-victims, profiles. 
(1997), entendido como "un vínculo social entre compañeros en el que un chico(a) o un grupo de chicos(as) abusa con cierta regularidad de su poder físico, social o psicológico sobre otro(s) u otra(s), al que someten o maltratan".

\section{Definición del problema}

El problema de la violencia y la agresividad se ha dado a lo largo de la historia de la humanidad; la agresividad intimidatoria entre los alumnos es un fenómeno muy antiguo, y el hecho de que varios alumnos sean blanco de las agresiones y el hostigamiento de otros niños se presenta descrito en obras literarias (Olweus, 1998). También los profesores lo viven directamente en su labor cotidiana.

En los planteles educativos se dan distintos comportamientos agresivos que traen como consecuencia unas condiciones no muy óptimas para el desarrollo y la formación normal de un individuo. Hay alumnos y alumnas que agreden a otro u otra no solo física sino psicológicamente, a partir de burlas, desprecio, groserías o rechazo, produciendo en las víctimas frustración, baja autoestima y otra clase de conflictos de personalidad, por el hecho de sentirse solos y aislados.

Pareciera que la única importancia que le dan las directivas de los colegios a la violencia escolar es cuando encuentran a dos alumnos peleando físicamente. ¿Qué se hace en estos casos? La solución más fácil es llevarlos a rectoría o coordinación disciplinaria, citar a sus respectivos padres, imponerles una sanción a los alumnos implicados y regañarlos o humillarlos. ¿Pero aprenden a no ser violentos? Los maestros y las maestras no indagan más allá de lo que han visto, y eso les es suficiente para catalogar a los alumnos como rebeldes e indisciplinados. Sin embargo, la violencia escolar ha sido un tema que atrae el interés de maestros y psicólogos, y por ello se han dedicado a estudiar la problemática para lograr establecer las posibles causas y consecuencias, y plantear alternativas de solución al conflicto.

El fenómeno de la violencia escolar es un problema que hasta ahora no se ha trabajado de manera rigurosa en nuestro país, y son pocos los estudios dedicados al tema, como los realizados por el IDEP (1999) en Vida de maestro. Violencia en la escuela, y el proyecto trabajado por Parra, González, Moritz, Blandón y Bustamante (1994), con el auspicio de la Fundación Fes. Otros trabajos investigativos han sido propuestos desde los proyectos "Ciudad educadora" (Villa y Moncada, 1998) y "Pléya- de" (Parodi, 1999). Según Funk (1997), existe un amplio consenso en relación con la idea de que no se puede hablar de un aumento general de la violencia en las escuelas. El planteamiento de Funk se podría cuestionar, ya que es un fenómeno de varias instituciones que se sale de las manos de los maestros y directivos, cuya única alternativa, al parecer sensata, es la exclusión del plantel educativo de los alumnos partícipes en comportamientos violentos.

Las conclusiones derivadas de esta investigación no tienen pretensiones de generalización, fuera de los límites que establece la caracterización de este grupo de niños sujetos de estudio.

¿Qué características en común (psicológicas, académicas) y qué condiciones contextuales (escolares, familiares) tienen los alumnos que son víctimas o victimarios en el fenómeno de la violencia escolar, en el nivel de tercero de primaria, con niños entre los 8 y 10 años de edad?

Esa es la pregunta base de esta investigación dada la particularidad que tiene el fenómeno de la violencia escolar, vista desde la perspectiva de la generación de perfiles, y articula una triple pertinencia: disciplinaria, interdisciplinaria y social. De esta manera, en relación con la pertinencia social, la violencia en las escuelas es un fenómeno que afecta a muchos de los actores de los planteles educativos, ya sean maestros, padres de familia o alumnos, y es necesaria una pronta intervención, puesto que el proceso de la educación en Colombia debe lograr una buena formación integral en niños y jóvenes que más adelante ingresarán a una sociedad que en la actualidad se ve afectada por los fenómenos de violencia. También constituye un punto clave para la contribución a la formación adecuada de niños con valores y buenos principios, además de valores formativos, y así mismo a la construcción de una sociedad más tolerante y con menos manifestaciones de violencia.

A partir de estudios sobre el tema de la violencia escolar se podrían plantear acercamientos a una realidad, con el fin de lograr y facilitar que los maestros adquieran un desarrollo de habilidades en el manejo y la prevención de la violencia y las intimidaciones entre los alumnos (Campart y Lindström, 1997). El fenómeno de la violencia escolar es un problema que podría llegar a tomar eventos inmanejables; por esta razón la Psicología, en el contexto educativo, es una herramienta para examinar el problema de la violencia escolar. 
En ese contexto educativo es necesario un proceso de trabajo interdisciplinario en el que actuarían en conjunto tanto profesionales de la pedagogía como psicólogos, para compartir experiencias y saberes que conllevarían a una complementación de la educación con buenos principios y valores de ciudadanos que formarán parte de nuestra sociedad colombiana.

El aporte en el ámbito interdisciplinario sería el de brindar conocimiento en el campo de la educación en los niveles tanto teóricos como prácticos, específicamente tratando de establecer las características comunes de los alumnos agresores y de alumnos víctimas. Como dice Carrasco, citado por Jácome (2003) en conferencia dictada en el curso de psicoanálisis y educación titulada "Una mirada a la relación educador-alumno", la educación tendría que ver, más allá de enseñar al que no sabe, con disponer al hombre para que pueda encontrarle sentido a la vida y, así también, con encontrarse con sentido en la vida.

\section{CESAR AUGUSTO SIERRA VARÓN}

Psicólogo de la Universidad Javeriana, Bogotá, con conocimiento y experiencia práctica en áreas como Psicología clínica y educativa. Docente de la Facultad de Ciencias Sociales, programa de Psicología, del Politécnico Grancolombiano, a cargo de las cátedras de Desarrollo y clínica infantil; Planeación y ejecución de talleres-conferencias para padres de niños y adolescentes. Psicoterapeuta de niños y adolescentes. Asesor pedagógico en la autoría de textos de educación preescolar, Editorial Creativa.

csierrav@poligran.edu.co

\section{COAUTOR}

\section{FELIPE ALFONSO ROJAS MONCRIFF}

Psicólogo. Coordinador del área educativa de la Facultad de Psicología de la Pontificia Universidad Javeriana, Bogotá.

felipe.rojas@javeriana.edu.co

\section{Fundamentación bibliográfica}

La violencia es un problema que actualmente enfrenta nuestro país. Es muy común ver situaciones en las cuales está involucrada la violencia o la agresión. Según el libro Violencia en la escuela (Instituto para la Investigación Educativa y el Desarrollo Pedagógico, IDEP, 1999), de la serie Vida de maestro que dirige la Alcaldía Mayor de Bogotá, la violencia se manifiesta de varias formas, por ejemplo: desconocimiento del otro al ignorarlo, o cuando su nombre nunca es pronunciado, pasando por el uso de la autoridad, el sometimiento de alguien abusando del conocimiento y de la edad, así como también al subyugar a los más débiles a una voluntad ajena a su deseos.

Existe un enfoque naturalista que le ha dado a este tema la etología, con lo cual se ha contribuido a desarrollar la creencia social de que la agresividad forma parte de la naturaleza humana (Ortega y Mora-Merchán, 1997). Esta ciencia estudia el comportamiento animal en su medio natural, frente a lo cual se han realizado estudios muy importantes para el conocimiento del hombre (Ledesma, 1980), y que pueden ofrecer explicaciones al comportamiento agresivo de este.

La mayoría de los etólogos elaboran conclusiones sobre la agresividad humana a partir de comportamientos observados en los animales. Sin embargo, las agresiones humanas alcanzan frecuentemente extremos mortíferos, que muy rara vez ocurren entre los animales (Kogan, 1994). Los estudios de Konrad Lorenz sobre la agresión eran biológicos y omiten la particularidad del hombre relacionada con su capacidad de crear símbolos y de manejarse con ellos (Rivarola, 1993). De hecho, la naturaleza exacerbada de la destructividad y la crueldad humanas pueden ser vistas como características, que por el lado del comportamiento distinguen al hombre de los otros animales (Freeman, 1977).

Autores como Albert Bandura y Richard Walters (1963) ya no se encuentran en una perspectiva etológica de la agresión y plantean que este tema debería ser visto desde la perspectiva del aprendizaje social, lo cual refiere a que en este tipo de aprendizaje se hace énfasis en la forma como se aprenden y mantienen los patrones de comportamiento agresivo. Estos autores investigan además la agresión que se aprende como medio para obtener determinados fines. Desde una perspectiva conductista ortodoxa, se diría que las conductas agresivas se adquieren principalmente mediante el reforzamiento positivo directo de las respuestas agresivas. Los adolescentes y niños podrían sufrir de acosamiento y humillación, razón por la cual más adelante, cuando son jóvenes adultos, reproducirían estos comportamientos agresivos si se les presenta la ocasión oportuna para ello, ya que se encuentran constantemente rodeados de modelos agresivos.

La psicología social, en especial la norteamericana, mediante estudios sobre las luchas raciales, los problemas de la microsociología y la influencia de la familia, entre 
otros, demuestra que un modelo social puede aumentar o reducir la agresión (Ledesma, 1980). La perspectiva social concede una gran importancia a la agresividad en el aprendizaje, lo que conlleva a una actitud optimista en relación con la educación de la agresividad, ya que el aprendizaje sociocultural puede conseguir tanto hábitos de violencia como también una inhibición habitual de la agresividad (Pastor, 1980).

Se puede observar que el tema de la agresividad ha sido tratado desde diferentes puntos de vista. De igual manera, las definiciones concretas de agresividad son prácticamente interminables y muy poco uniformes (Funk, 1997). Sin embargo, para lograr una generalización, la agresividad estaría definida frente a su fin de lesionar a otro organismo o al propio, pero sería necesario añadir a lo anterior la intención se producir un daño, destruir, contrariar o humillar. La agresividad es entendida como el término para designar la tendencia o disposición inicial que daría lugar a la posterior agresión, entendida esta última como el término que debería utilizarse para designar un acto en sí, un acto palpable y efectivo (Fernández, sf).

Sin embargo, a pesar de que existen quienes sostienen perspectivas de origen social con respecto a la agresividad, también están quienes piensan en la agresión como una carga del nivel biológico insuperable; y otros que la atribuyen al ambiente cultural. Pero es importante recalcar que ambos enfoques, tanto el biológico como el cultural, no se excluyen. La agresión es vista como parte del equipo básico del hombre, pero también se encuentra condicionada, exacerbada u orientada culturalmente (Rivarola, 1993).

Se da paso al tema de la "violencia" y a las diferentes perspectivas desde las cuales ha sido abordado, ya que, al igual que en el tema de la agresión, son múltiples las teorías que se han postulado al respecto. No se encuentra tampoco un consenso sobre la definición del término "violencia" como tal. La violencia es claramente un acto de agresión contra otra persona. Si se está hablando de violencia entre personas, quiere decir que en un conflicto hay implicados más de un individuo. Se presenta entonces una relación combinada víctima-ofensor. La mayoría de las veces la violencia se encuentra dirigida hacia una persona o un grupo particular (Gunn, 1976). Asimismo, parece difícil tener que aceptar que solamente es violento el acto que culmina con algún daño físico o hasta el de la muerte, y con ello se excluyen otros tipos de agresión de orden más psicológico y simbólico (Camargo, 1997). Por otro lado, Planella (1998), citado por Fernández (sf), considera la violencia como una situación en la cual dos o más individuos se encuentran en una confrontación en la que una o más personas salen perjudicadas por agresión física o psicológica. La violencia tiene lugar en el momento en que, dada una confrontación de intereses, uno de los protagonistas se coloca en un lugar de dominio dejando al otro en un lugar de impotencia que lo obliga a la sumisión. El esquema psicológico de la violencia tendría que ver con un modelo de dominiosumisión (Ortega, 1997).

El mecanismo de la violencia se presenta en todos los niveles del sistema social, en todos los países y todas las culturas. Los medios de comunicación nos presentan permanentemente manifestaciones de violencia como masacres, secuestros, encuentros armados entre soldados, terrorismo, maltrato a niños, etc. Se observa entonces que es un tema que se presenta en la cotidianidad y que sus manifestaciones son múltiples (Blanco, Docal y Villamizar, sf).

El desarrollo humano siempre va a encontrarse influido por la crianza y educación proporcionadas por el ambiente y por los adultos experimentados en una determinada sociedad. En los primeros años se depende enteramente de otras personas; se aprendería a amar a los demás en etapas posteriores de nuestro desarrollo, pero si en los primeros años de vida este aprendizaje resulta deficiente, la habilidad de amar resultaría incompleta (Gunn, 1976). Este autor hace referencia a la gran influencia que tiene el medio como método de aprendizaje para cierto tipo de comportamientos. Así se podrían reforzar los comportamientos violentos en el ser humano. También menciona como uno de los mecanismos para la adquisición o formación de comportamientos violentos, el del "aprendizaje por imitación", que consiste en un proceso humano educacional muy poderoso. Muchos criminólogos sostienen la teoría de que el adulto cruel y violento ha adquirido tales características de sus padres.

Una de las caras de este tema es la intrafamiliar. La familia puede ser el vehículo para descargar las frustraciones y limitaciones sociales del individuo, quien la utiliza para ejercer poder sobre los débiles. En el espacio familiar también se dan formas de violencia subjetiva, lo cual conlleva a que se reproduzcan esquemas que generan violencia en otras instancias sociales (Blanco, Docal y Villamizar, sf). 
En el estudio de McCord, McCord y Howard (1961) sobre los correlatos familiares de la violencia en niños delincuentes, refieren el hallazgo en su estudio de que, aunque se esperaba encontrar que niños con padres violentos presentarían a su vez bastantes conductas de tipo violento, dicha tendencia no alcanzaba a ser significativa. Sin embargo se encontró que niños cuyos padres emplean métodos no violentos para disciplinarlos y razonan con ellos, presentan conductas menos violentas que los niños cuyos padres emplean métodos más punitivos y violentos para la crianza de sus hijos, aunque en una proporción significativamente más baja. De esta forma la televisión cumpliría un papel importante ya que este es un medio por el que se transmite mucha violencia que se encuentra altamente difundida en las comunidades, con su alto poder de influencia sobre un elevado número de personas al mismo tiempo (Gunn, 1976). Esta gran influencia de la televisión en los juegos de los niños se puede observar cuando juegan a matar (rambos, karatekids, o en los roles de las pandillas de barrio) y que se interpretan perfectamente en las horas de recreo (Valdés, 1991).

Si se realiza una reflexión al respecto, este es el mensaje típico de innumerables ficciones cinematográficas o televisivas en las que se suelen asociar superioridad física, justicia y éxito, actuando como refuerzo de la violencia (Kogan, 1994). Sin embargo, el aprendizaje vicario de la violencia que es difundido por los medios no es suficiente por sí solo para dar cuenta del aumento de las conductas violentas en la sociedad (Ledesma, 1980). Asumir la violencia con la manera particular que tiene para anudarse en la experiencia vital de cada persona, conduce directamente a interrogar a las instancias que pueden llegar a mediar entre lo que podría llegar a considerarse como individual o como grupal y colectivo (Alvarado, 1995). De esta forma, la violencia es concebida como un fenómeno que trasciende la simple conducta individual para convertirse en un proceso interpersonal, porque afecta al menos a dos protagonistas: quien la ejerce y quien la padece (Ortega y Mora-Merchán, 1997).

En un contexto mucho más específico, el fenómeno de la violencia también se presenta en muchos de los hogares de nuestro país. Existen familias donde se presentan condiciones y dinámicas basadas en métodos jerárquicos y autoritarios que cobran como víctimas a los más débiles, como son las mujeres y los niños. Se debe identificar la violencia y crear los mecanismos, competencias y responsabilidades para combatirla (Armenta, 1999). Tanto la escuela como la familia son contextos en los cuales se presentan manifestaciones de violencia y maltrato infantil, ya sean estos comportamientos violentos entre pares o en dirección adulto-niño. Castigos extremadamente estrictos, gritos, insultos, respuestas déspotas y la sobreprotección que limita la autonomía en los menores son formas de maltrato infantil imposibles de medir, pero que se presentan diariamente en los hogares y en los centros educativos (Blanco, Docal y Villamizar, sf). Es así que se le da una gran importancia al contexto educativo en el que el niño o la niña también se desenvuelve en sus relaciones sociales y en el que tiene que convivir con otros niños, y en el que se generan conflictos entre ellos que en ocasiones llevan implícitas vías de violencia y agresión. Este fenómeno de violencia puede adoptar niveles inmanejables con niños bastante agresivos que maltratan con mucha frecuencia a otros compañeros que consideran más "débiles".

La escuela es tomada como una de las instituciones sociales más importantes para el desarrollo de la sociedad, ya que en ella tienen lugar procesos de instrucción y socialización, que son, al igual que los que se producen en la familia o en el contexto cultural, responsables de la integración social de los jóvenes. Pero los propósitos que tengan los diferentes centros educativos en la formación de niños y jóvenes que se desenvolverán más adelante en una sociedad no se lograrán solamente a partir de la transmisión del aprendizaje de contenidos curriculares, sino que también se requiere de una compleja red formada por los sistemas de relaciones personales y los procesos psicosociales de convivencia y comunicación, que vayan articulando los procesos instructivos y socializadores (Ortega, 1997).

Abordar el tema de la violencia escolar en Colombia refleja ante todo un compromiso por parte de instituciones y de investigadores, en una realidad social compleja que afecta a nuestro país, definido por algunos como uno de los más violentos, a la vez que como un país de contradicciones: de armonía y maltrato, de paz y de guerra, de alegría y de tristeza (Mejía, 1997).

Angulo (2003), en su artículo "Violencia escolar, un fenómeno mundial", menciona que durante los últimos años la preocupación aumenta por el creciente registro de hechos violentos y conflictos en las instituciones educativas. Se puede suponer que las formas de expresión de dicho fenómeno varían de acuerdo con las condiciones sociales de cada centro educativo y al entorno social que rodea la institución. Es importante tener en cuenta que la sociedad convive con acciones violentas en todos sus contextos. Pero penetrar en el tema de la 
violencia escolar no es tan fácil, por el hecho de que plantea una gran ambivalencia: no es un tema que se encuentre muy bien precisado; su uso es generalizado, muy amplio y sin especificaciones; y porque en la escuela la problemática de la violencia es trabajada muy escasamente, lo que conlleva al no reconocimiento de su existencia y a no reflexionar sobre ella. Es difícil delimitar el problema porque no todo lo que pasa en la escuela es violencia, pero tampoco se puede pensar que allí no pasa nada violento (Camargo, 1997).

Se considera de igual importancia el papel que interpretan los agentes de socialización, tales como los medios de comunicación, así como "la calle". Se debe anotar, en primera instancia, que para muchos niños y jóvenes los medios de comunicación y el contexto callejero cumplen papeles sustitutos en el tiempo en que los padres no están con ellos (Peláez, 1991). Ardila (1999) menciona que el "parche" para los muchachos significa la posibilidad de encontrar el afecto y el amor que por lo general no encuentran en los miembros de sus familias. Tales amigos que conforman el "parche" se convierten entonces en la fortaleza del pandillero. Al ingresar a un grupo social pandillero, sus integrantes adquieren varios cambios en su forma de ser y hasta en su personalidad habitual. Pueden llegar a adquirir hábitos en sus comportamientos que se trasladan a otros contextos como el escolar.

En los niños se suelen presentar conductas de imitación y, por consiguiente, imitan comportamientos considerados como buenos y como malos. Es así entonces como es posible encontrar alumnos de cierta edad con tendencias malas e indisciplinadas en la escuela; por ejemplo, el hecho de hacer justicia por su cuenta: si el niño observa conductas por influencia de los medios, así como también del ambiente callejero en el que se desenvuelve, se puede evidenciar que, ante un conflicto con otro compañero, ya no será necesario acudir al maestro para plantearle su caso, sino que él mismo ejecuta la acción y se cobra el mal que le causaron (Parra, González, Moritz, Blandón y Bustamante, 1994).

Los actos violentos se encuentran inmersos en un gran sistema de relaciones interpersonales, en el cual se encuentran presentes -y configuran el campo educativo- las emociones, los sentimientos y los aspectos cognitivos, incluyendo las situaciones familiares de cada alumno y el ámbito social en que se encuentra la escuela. Pero el problema surge cuando se dan conflictos y su resolución se presenta por medio del ejercicio de la autoridad, del castigo, etc., provocando un clima en el aula con mucha tensión, que muchas veces los maestros no saben resolver (Ochoa, 2000). Casos más graves se pueden encontrar en eventos en los que la violencia escolar adopta la forma de tiroteos en la escuela (school shootings), problemática que se ha divulgado a partir de ciertos casos ocurridos en Estados Unidos y Europa (Ruiz, 2002).

Hasta hace poco tiempo la ciencia no tenía la oportunidad de ofrecer datos actualizados sobre la trascendencia del problema, en especial sobre el aumento de la violencia escolar que los medios de comunicación destacaban de forma insistente (Funk, 1997). En la actualidad, aunque no son muy reconocidos, se han realizados estudios investigativos en relación con el tema de la violencia escolar; es así como Ortega y Mora-Merchán (1997) citan un estudio llevado a cabo en España sobre el fenómeno de la intimidación y victimización entre compañeros, realizado en 1989, en el cual los investigadores diseñaron un cuestionario para sus objetivos, cuyos resultados mostraban que aproximadamente el $17 \%$ de los escolares encuestados admitían haber sido intimidadores y el 17,2\% víctima de forma muy frecuente. Esto se relaciona con el término "matoneo" entendido como cualquier tipo de maltrato que ejerce una persona sobre otra convirtiéndola en sumisa. El tipo de matoneo que más ha llamado la atención es el maltrato ejercido por unos niños hacia otros, aunque no sea esta la única forma de matoneo. El matoneo implica agresión y muchos "matones" adoran sus actividades agresivas porque obtienen alguna satisfacción de ellas (Yorke, 1997). El matoneo se reconoce "cuando un niño grande y fuerte golpea a un niño más pequeño" (Marland, 1997: 227); es el abuso del más fuerte hacia el más débil, con la consideración de que no siempre ser "fuerte" significa mayor fuerza física, sino que también está relacionado con el sentido de poder, por lo general ejercido por el placer que le da a quien lo ejerce (Yorke, 1997).

Dan Olweus introduce un concepto que trae un significado similar al de "matoneo", utilizado en la actualidad y muy común en las investigaciones realizadas sobre violencia escolar, que es el de "bullying", término con el cual se hace referencia a conductas o situaciones de acoso e intimidación de un alumno o de alumnos hacia otro u otros. Olweus (1993), citado por Ortega y MoraMerchán (1997), define la victimización como una conducta que conlleva persecución física o psicológica que realiza un alumno a otro, a quien elige como víctima en repetidos ataques. Esta acción intencionada coloca a la víctima en una situación de la que difícilmente puede salir por sus propios medios. 
Los alumnos protagonistas tienen ciertas particularidades comunes, tanto para las víctimas como los agresores; por tal razón, se presentan ciertas características típicas que identifican de alguna manera a estos protagonistas de la violencia escolar, según lo plantea Olweus (1998).

Las víctimas típicas son por lo general alumnos más ansiosos e inseguros que los demás, que cuando se sienten atacados reaccionan normalmente llorando y alejándose. Padecen de baja autoestima con una opinión negativa de sí mismos y de su situación. Con frecuencia se consideran fracasados, estúpidos y avergonzados. En los colegios se les observa solos y aislados, casi no tienen amigos en su clase y no muestran conductas agresivas ni burlonas, de lo cual se infiere que el acoso y la intimidación no se pueden explicar por las provocaciones que las propias víctimas pudieran hacer a sus compañeros. También suelen tener una actitud negativa frente a la violencia y el uso de medios violentos. El autor clasifica este grupo de víctimas como pasivas o sumisas: niños que no responderán al ataque o al insulto. También se caracterizan por un modelo de ansiedad y de reacción sumisa, combinada con una debilidad física.

La otra categoría que Olweus propone para las víctimas es la de provocadoras, caracterizada por una combinación de modelos de ansiedad y reacciones agresivas. Ellas suelen tener problemas de concentración y se comportan de forma que causan irritación y tensión a su alrededor. No es raro que su conducta provoque a algunos alumnos de su clase. Al igual que las víctimas pasivas, también pueden ser más débiles físicamente que sus compañeros. Además, suelen ser malgeniados, intentan pelear o responder cuando se les ataca o se les insulta, pero normalmente de forma ineficaz. Pueden ser hiperactivos, inquietos, dispersos y ofensivos y de costumbres irritantes, y es posible que provoquen el disgusto activo de los adultos, incluidos los profesores, y pueden intentar agredir a otros escolares más débiles.

De otro lado, lo que suele caracterizar a los agresores típicos, tomando como referencia lo que este mismo autor propone, es su belicosidad con los compañeros, aunque a veces también se muestren de la misma forma con los adultos, tanto profesores como padres. En general, tienen una actitud de mayor tendencia hacia la violencia y el uso de medios violentos que los demás alumnos. Suelen ser impulsivos y tienen una necesidad imperiosa de dominar a los demás y poca empatía con las víctimas de sus agresiones. Con frecuencia tienen una opinión positiva de sí mismos, son físicamente más fuertes que sus víctimas y no padecen de baja autoestima. También es necesario mencionar que existen alumnos que, aunque son agresivos y participan en las intimidaciones hacia los otros, normalmente no toman la iniciativa; a estos alumnos, el autor los clasifica como "agresores pasivos, seguidores o secuaces". Son alumnos que se suelen rodear de un grupo de compañeros que los apoyan y parecen simpatizar con ellos. Sienten una necesidad imperiosa de poder y de dominio, parecen disfrutar cuando tienen el control y necesitan dominar a los demás. Además, si se consideran las condiciones familiares en las que se encuentran estos niños, se puede suponer que han desarrollado cierto grado de hostilidad hacia el entorno, y tales sentimientos pueden llevarlos a sentir satisfacción cuando producen daño y sufrimiento a sus compañeros. A su vez, se encuentra el componente del beneficio que consiguen con sus comportamientos, ya que los agresores con frecuencia obligan a sus víctimas a que les den dinero, entre otros elementos. Son físicamente eficaces en los juegos, los deportes y las peleas; se enfadan fácilmente; son poco tolerantes a la frustración y les cuesta adaptarse a las normas y aceptar las contrariedades. Con los adultos suelen tener una actitud hostil, desafiante y agresiva; no son ansiosos ni inseguros. Su rendimiento académico puede ser normal o estar por debajo o por encima del promedio en los grados de primaria.

En la escuela misma se pueden observar conductas de tipo violento, tales como muertes, amenazas o "boleteos", así como también comportamientos agresivos hacia otros. Es importante empezar a asumir la violencia como fenómeno de importante reflexión en la institución educativa (Camargo, 1997).

\section{Objetivos}

\section{Objetivo general}

Describir y extraer características de los niños agresores y de los niños víctimas en el fenómeno de la violencia escolar, con el fin de generar perfiles de cada uno de ellos (agresores y víctimas), con alumnos de grado tercero de primaria de un centro educativo distrital en Bogotá.

\section{Objetivos específicos}

- Identificar y describir los diferentes tipos de violencia y victimización que se dan entre alumnos del grado tercero de primaria 
- Determinar las condiciones en que se dan los diferentes comportamientos violentos

- Analizar el contexto familiar, escolar, personal e interpersonal de los niños agresores en el centro escolar

- Analizar el contexto familiar, escolar, personal e interpersonal de los niños que se convierten en víctimas de agresiones con más frecuencia

\section{Categorías}

Las categorías que iluminaron la presente investigación se describen a continuación.

\section{Agresores o agresoras}

Son alumnos o alumnas que suelen participar como perpetradores en actividades como la de gastar repetidamente bromas desagradables a otros compañeros; pueden ser físicamente más fuertes, eficaces en los juegos, deportes y las peleas; sienten una necesidad imperiosa de dominar y subyugar a otros niños; se imponen mediante el poder y la amenaza; se enojan con facilidad; son impulsivos y tienen baja tolerancia a las frustraciones; y les cuesta adaptarse a las normas y aceptar las contrariedades. Con los adultos suelen ser hostiles, desafiantes y agresivos, no son muy ansiosos ni inseguros, y casi no tienen simpatía por los alumnos que escogen como víctimas, entre otras características.

\section{Víctimas}

Son niños o niñas objeto de agresiones y hostigamiento por parte de otros compañeros. De esta misma categoría se pueden desprender dos subcategorías, teniendo en cuenta la clasificación que propone Olweus (1998) para determinar dos posibles clases de víctimas.

Víctimas pasivas o sumisas: Son alumnos ansiosos e inseguros; suelen ser cautos, sensibles y tranquilos; cuando se sienten atacados normalmente reaccionan llorando y alejándose; padecen de baja autoestima y con una opinión negativa de sí mismos y de su situación; con frecuencia se consideran fracasados, estúpidos, avergonzados y sin atractivo físico. En la escuela se les observa como niños aislados y solos; normalmente no tienen ni un solo buen amigo; no muestran conductas agresivas ni burlonas hacia otros compañeros; y suelen tener una actitud negativa ante la violencia y el uso de medios violentos. No responden ni a los insultos ni a los ataques que les hagan. En cuanto a la relación con sus padres, son niños muy apegados a ellos, en especial a sus madres.

Víctimas provocadoras: Son alumnos que se caracterizan principalmente por una combinación de modelos de ansiedad y de reacciones agresivas. Se comportan en forma que causa irritación y tensión a su alrededor; algunos de ellos pueden caracterizarse como hiperactivos. Sus conductas pueden provocar a muchos alumnos de su clase, posiblemente pueden provocar el disgusto activo de los adultos. También suelen ser más débiles físicamente que sus agresores; pueden tener una opinión negativa de sí mismos; pueden ser malgeniados; e intentan pelear o responder cuando los atacan o insultan, aunque sus resultados no sean eficaces.

\section{Violencia física}

Entendida por lo que Mejía (1997) describe como cualquier forma de agresión infligida por parte de una persona hacia otra, producida por el uso de la fuerza física no accidental. En esta categoría se exploraron los comportamientos de los niños en torno a su agresión hacia los demás, tales como los puños, los empujones, las cachetadas y halar el cabello, entre otros.

\section{Violencia psicológica o emocional}

Según Mejía (1997), este tipo de violencia se manifiesta de diferentes maneras, entre ellas mediante la ausencia de afecto, el desconocimiento del otro, la humillación ante los demás, la burla, el desprecio, los insultos, el regaño y el uso de la autoridad o de algún rango de superioridad, dados por cualquier aspecto, ya sea físico o intelectual, para amedrentar o someter.

\section{Conductas de acoso y amenaza entre esco- lares (victimización)}

Existen ciertos niños que son objeto de agresiones y hostigamientos con bastante frecuencia por parte de otros compañeros. Siguiendo los planteamientos de Olweus (1993), citado por Ortega y Mora-Merchán (1997), se entiende entonces la victimización como una conducta que conlleva persecución física o psicológica realizada 
por un alumno contra otro, a quien elije como víctima en repetidos ataques.

\section{Variables en la escuela}

La relación que tiene cada uno de los participantes en la violencia escolar (víctimas, agresores), tanto con sus compañeros y maestros, así como la forma de actuar y comportarse en la escuela, entre otros aspectos. También fue importante el análisis del rendimiento académico de estos niños seleccionados como víctimas y agresores, ya que, desde la teoría de Olweus (1998), por lo general los alumnos que son víctimas frecuentes presentan un deterioro en el rendimiento académico muy notorio. De igual forma lo fueron las perspectivas que tienen otros compañeros y los profesores, exploradas por medio de las entrevistas semiestructuradas.

\section{Variables familiares}

Se indagó sobre las relaciones y dinámicas que se mueven en las familias de cada uno de los sujetos participantes de la investigación, buscando un contacto directo con la propia familia (la mamá de los niños). De esta forma se logró conocer cómo es el ambiente familiar de los niños víctimas y de los niños agresores, las relaciones con sus padres, con sus hermanos, y si existen o no comportamientos violentos en la vida cotidiana.

\section{Representación social de la violencia en la escuela}

La representación social hace referencia a un proceso psicosociológico, el cual refleja las formas específicas como se construye la realidad social, así como las relaciones interpersonales.

\section{Método}

\section{Diseño}

El presente trabajo de investigación se desarrolló basado en un diseño metodológico descriptivo, con el cual se identificaron los posibles rasgos característicos típicos de los niños agresores y de los niños víctimas en la violencia entre escolares, con el fin de generar perfiles de cada uno de ellos: niños y niñas del grado tercero de primaria de un centro educativo distrital en Bogotá. Así mismo, queda inscrito en el campo de la investigación cualitativa. Como lo dicen Gómez y Ramírez (2000), la organización del trabajo cualitativo se caracteriza por ser flexible en su estructura y dinámico en su aplicación, estableciendo como condiciones básicas la contextualización del mundo de la realidad cotidiana, en este caso la violencia escolar, así como "un abordaje teórico conceptual”.

La investigación etnográfica fue dirigida a analizar esta problemática, mediante la generación de perfiles de los niños agresores y de los niños víctimas, puesto que al realizar una observación de las dinámicas que se juegan en la institución educativa -que producen relaciones de tipo violento e inician el fenómeno de la victimización e intimidación entre escolares-, se logró una comprensión de los diferentes casos de los niños partícipes de este fenómeno, mediante la generación de sus respectivos perfiles.

\section{Participantes}

Para este trabajo investigativo se trabajó con un grupo de alumnos de grado tercero de primaria de un centro educativo de Bogotá, entre 8 y 10 años de edad. Además de estos niños, se tuvo muy en cuenta la participación de los maestros titulares, quienes colaboraron con la información de los niños más agresivos y violentos, y de los niños víctimas más frecuentes de sus respectivos cursos. Sin embargo, para la generación de los perfiles de agresores y de víctimas se trabajó con seis alumnos involucrados en la violencia escolar (tres niños más agresivos del salón; una niña, la más agresiva de su clase; y dos niños que son víctimas). Los niños con los cuales se trabajó pertenecen al estrato socioeconómico 2.

El criterio de selección de los participantes se basó en aspectos como el tipo de muestreo no probabilístico de tipo reputados, ya que, una vez que los maestros y las maestras conocieron el tema de la observación, le recomendaron al investigador el grupo de alumnos que presentan características de personas violentas y agresivas, y de las que caen en el papel de víctimas frecuentes. Esto último implicó un procedimiento de selección de tipo de los casos excepcionales (Goetz y LeCompte, 1988).

\section{Instrumentos}

Entre las estrategias de recolección de información con el grupo de estudio se consideró como principal la de 
la observación naturalista, que consiste en focalizar totalmente la atención sobre ciertos sectores específicos de la realidad física y social; en este caso, los comportamientos violentos presentados en la escuela por parte de los miembros (alumnos) que allí participan de manera intencional, registrando los elementos constitutivos y la dinámica de la situación (Taylor y Bogdam, 1996). La observación fue de tipo no participante.

La primera parte del proceso de investigación fue la observación de campo y la recolección de información en diarios de campo. Esto permitió conocer el grupo y el ambiente que se vive en la institución durante varias jornadas escolares en las horas de la mañana, teniendo en cuenta su dinámica, el contexto institucional, la manera en que se llevan a cabo las relaciones interpersonales entre los alumnos, etc. Una estrategia que se consideró para llegar a una validación de las observaciones fue la de la triangulación de información recolectada en los diarios de campo, con la información que ofrecieron los maestros de estos alumnos. De esta manera se corroboraron los datos recolectados con el conocimiento más profundo que tienen los maestros titulares de los niños.

En el diario de campo se fueron registrando las escenas pertinentes para el tema de observación (considerando las categorías como guías de observación). La información recogida con este instrumento fue abordada con notas condensadas y notas expandidas, personales y metodológicas. Otro de los instrumentos utilizados para la recolección de información fue la entrevista semiestructurada, que constituye un método de investigación y descubrimiento (Gómez y Ramírez, 2000). La entrevista se realizó a los sujetos (niños y niñas) seleccionados como los más agresivos y los que se convierten en víctimas frecuentes, así como también a los profesores y compañeros, con el fin de indagar sobre los posibles aspectos y rasgos característicos que ellos perciben de estos sujetos. También fue realizada a sus familiares (mamás).

Dicho instrumento, en el proceso de investigación, fue presentando para validación de jueces antes de su aplicación. El formato inicial se presentó para su respectiva validación a dos jueces expertos, docentes del Departamento de Psicología de la Pontificia Universidad Javeriana, que trabajan el área de investigación y metodología.

\section{Procedimiento}

En primera instancia se solicitó el permiso correspondiente a la institución educativa para llevar a cabo la investigación. Con posterioridad se hizo un acercamiento con la población que participaría en el estudio. De esta manera se llevó a cabo una primera parte del estudio, la cual se basaba en la observación naturalista de la dinámica escolar en general, luego se fueron seleccionando los alumnos que participarían en el estudio como agresores y víctimas para extraer sus respectivos perfiles. En la segunda parte del estudio, la observación se focalizó únicamente en los comportamientos de los niños seleccionados como agresores y víctimas más frecuentes. Así mismo, la información iba siendo recolectada en los diarios de campo.

Luego de la recolección de la información se dio inicio al proceso de análisis de los resultados de acuerdo con las categorías, para que de esta forma se determinaran las características típicas de los niños agresores y de los niños víctimas. Finalmente, se procedió a dar una interpretación comprensiva de los datos recopilados en los dos momentos de la investigación, para hacer un análisis descriptivo y lograr el objetivo principal de la investigación: presentar y describir rasgos característicos de los niños agresores y las víctimas con el fin de elaborar perfiles de cada uno de ellos.

\section{Resultados}

Para producir un análisis adecuado de los resultados se llevó a cabo una división por categorías con su respectiva descripción, estableciendo los siguientes puntos que ilustran de forma sistemática los resultados obtenidos:

\section{El agresor o la agresora}

Son varios los rasgos que caracterizan a este tipo de niños y niñas, entre los cuales se nombran los siguientes: son alumnos que suelen participar como perpetradores en actividades como la de gastar repetidamente bromas a sus compañeros y ponerles apodos. Los apodos hacen referencia a una distorsión cómica del nombre de sus víctimas, y toman rasgos físicos de la víctima para burlarse de ellos. También pueden burlarse de sus víctimas cuando se presenta alguna situación bochornosa o penosa que dejan a estas en ridículo frente a sus demás compañeros (Sierra, sf). Las insultan con groserías y palabras soeces, transmitidas a la víctima de 
forma directa. Menosprecian y ridiculizan a otros compañeros. Debido a su condición de agresores, peleones, indisciplinados y "fuertes del salón", estos agresores pueden desafiar a otros compañeros, buscarles pelea en cualquier momento o defender a otros compañeros que no emplean generalmente conductas violentas, y que se encuentran en una situación de hostigamiento por parte de otro niño. Tal condición de agresores, peleones y fuertes del salón puede ser otorgada por otros niños de su clase, quienes los señalan como tales y les tienen miedo; o también por parte de los mismos agresores, ya que pueden referirse a ellos mismos como "el niño que maltrata a sus demás compañeros”.

Asimismo resaltan por su agresividad. Son alumnos que amenazan y les dan órdenes a otros niños, con lo cual los agresores quedan en una posición que deja a su víctima en una actitud de sumisión por temor o miedo a las amenazas. En los juegos se puede observar que son los que por lo general colocan las reglas. Se pueden imponer mediante el poder, la fuerza física o las amenazas, y de esta manera conseguir lo que se proponen. Se enojan con facilidad y son impulsivos. En relación con la violencia física se observan formas de victimización y hostigamiento por medio de golpes, puños, empujones y patadas, entre otros. En lo que hace referencia a la violencia psicológica o emocional se observan formas de victimización y hostigamiento mediante apodos, amenazas, formas de subyugación a los otros niños, menosprecios y conductas amedrentadoras, desafiantes o intimidatorias. En relación con su apariencia física se observa que los niños agresores no necesariamente son más fuertes físicamente que sus víctimas, ya que ellos también pueden victimizar a otros niños que incluso son más grandes y con mayor contextura física que ellos. Estos comportamientos de enfrentamiento y desafío con violencia física y psicológica a niños con apariencia física más fuerte que los agresores se da más entre los niños que en la niña clasificada en esta categoría. Esta niña por lo general presenta enfrentamientos, hostigamientos, amedrentamientos, uso de violencia física y psicológica o emocional, con niñas o niños que se encuentran en su mismo nivel de estudios.

La forma que emplean estos niños de victimizar a otros es individual, en general, y casi nunca victimizan a sus compañeros en grupo. De igual forma no se evidencia una gran diferencia en la frecuencia a la hora de utilizar más las agresiones físicas o las psicológicas. Se observa su gusto por fomentar peleas entre compañeros y el gusto por juegos que impliquen violencia física, ya que ellos siempre van a estar presentes cuando se trata de participar en los juegos de pelea, karate, rambo, tae-kondo, o el juego de pelear y golpearse con sus respectivos sacos de los uniformes. Les cuesta adaptarse a las normas y asumir las reglas que se fijan como parámetros del sistema escolar; por tal razón son niños considerados como indisciplinados, inquietos o hiperactivos. Con los adultos sí pueden mostrar una actitud desafiante y pueden ser hostiles y agresivos, aunque no reaccionan con violencia física. Estos niños agresores no tienen una víctima específica que se convierta en objetivo exclusivo de sus manifestaciones de violencia física y psicológica, ya que puede ser cualquier niño, bien sea de su curso, de otros cursos, o incluso de niños de niveles superiores al de ellos.

Por lo general se observa que no se presentan lazos de amistad muy estrechos entre las "víctimas" y los "agresores". La manera como estos niños entablan sus relaciones con los demás no es determinada, ya que pueden compartir de igual forma con todos los alumnos integrantes de su salón de clases, sintiéndose cómodos al participar en cualquier grupo. Poco les gusta compartir juegos con las niñas y prefieren permanecer más tiempo y compartir juegos con niños que se clasifican en esta categoría en particular. Tampoco les importa permanecer solos. En muy pocas ocasiones se convierten en víctimas; sin embargo, la forma de reaccionar por parte de estos niños cuando se sienten atacados o maltratados por otros niños es de forma agresiva. No tienen bajos niveles de autoestima y tienen buena opinión de sí mismos. En clase difícilmente se concentran, siempre están molestando a sus compañeros, se distraen con facilidad, interrumpen las clases continuamente, y en las clases al aire libre, como la de educación física, no manejan normas, siempre están peleando, les pegan a sus compañeros y en los descansos buscan hacer diabluras. El rendimiento académico de estos niños se ubica dentro del promedio.

\section{Víctima}

Es necesario mencionar que los alumnos agresores pueden agredir y victimizar de igual manera a cualquier niño sin importar quién sea. Las víctimas parecen más débiles que sus agresores, no les gusta participar en juegos en los cuales haya únicamente niños, como fútbol o los juegos de pelea. Comparten la mayoría del tiempo con niñas y en estos grupos pueden a veces dominar, mandar e incluso agredir a quienes son más débiles que ellos, en este caso las niñas. Cuando es- 
tos niños se sienten atacados, ofendidos o maltratados por otros, sus reacciones típicas pueden ser: colocar la queja ante la profesora, llorar, ignorar la agresión o patalear; en otras ocasiones pueden reaccionar de igual forma, es decir, de forma agresiva y enfrentarse ante su agresor y crear una disputa, así sea de manera verbal, y asumir el papel de agresores. Sin embargo, estos enfrentamientos se presentan en muy raras ocasiones, ya que en términos generales estos niños no reaccionan ante las ofensas de manera agresiva; por el contrario, optan por asumir una posición pasiva ante las agresiones y no se suelen defenderse activamente de estos ataques. Son alumnos más inseguros que los demás. En varias ocasiones, las agresiones hacia estos niños también pueden provenir de las niñas.

Sobre la clasificación que realiza Olweus (1998) con respecto a las víctimas, es decir, víctimas pasivas o sumisas y víctimas provocadoras, es necesario resaltar que tal clasificación se ha considerado de acuerdo con los comportamientos característicos de estos niños víctimas, ya que estos en ocasiones pueden asumir el papel de víctimas pasivas y en otros el de víctimas provocadoras. Así mismo, pueden presentar algunos rasgos característicos de cada uno, y en otras oportunidades no. Tienen un mejor amigo, y lo particular de estos mejores amigos es que son víctimas. No agreden a los compañeros que los victimizan con frecuencia, clasificados en la categoría de "agresores". Además, suelen tener una actitud negativa ante la violencia y el uso de medios violentos. Tienen en cuenta otras alternativas de solución ante los problemas entre compañeros, como "hablar o dialogar". Estos niños tienen baja autoestima al no poder relacionarse con otros niños. También les da miedo plantear sus ideas y exponer lo que piensan ante el grupo, así sepan que tienen la razón. Sin embargo, no tienen una opinión negativa de sí mismos ni de su situación. Pueden presentar una combinación de modelos de ansiedad y de reacciones agresivas, ya que, en muy pocas ocasiones, sí pueden reaccionar de manera agresiva cuando se sienten atacados por otros niños, aunque sus resultados no sean eficaces, debido a que son poco agresivos y sus contrincantes lo pueden ser en mayor grado. Pueden causar irritación y tensión a su alrededor, tanto al profesor como a otros alumnos, y por eso en ocasiones son víctimas de agresiones provocadas por ellos mismos.

No presentan características de desatención, no son inquietos ni les falta concentración, ni tienen rasgos de niños depresivos, aunque en ocasiones se sientan solos, aislados y tristes. Su rendimiento académico es bueno. No se observa en ellos desmotivación por asistir a la escuela, ni miedo por acudir a esta por temor a una posible victimización.

\section{Violencia física}

Se hace referencia aquí a los comportamientos que implican cualquier forma de agresión infligida hacia otra persona haciendo uso de la fuerza física no accidental. Se pueden encontrar comportamientos como pegar puños, halar del cabello, morder, pellizcar, empujar, votar al otro al suelo, pegar patadas, pelear, pegar con un lápiz o pegarle rodillazos en el trasero a otro compañero. Existen juegos que implican violencia física como el boxeo, la guerra de caballitos, el karate, la pelea o los cogidos. En estos juegos siempre participan alumnos agresivos que muestran claramente su gusto por tales juegos. Otros comportamientos violentos son: correr para pegarle una palmada en la cabeza, en la espalda o en el trasero a otro compañero, agarrar por el cuello a alguna víctima para dejarla sometida y luego golpearla, golpearle la cabeza a alguien contra la pared, tirarle tierra en los ojos, en la cabeza o en la boca, lanzarle objetos cortopunzantes como lápices o tijeras, y morder. En juegos como el fútbol se observan conductas agresivas como pegar patadas, puños, empujar, golpear con los codos y hacer zancadilla a otros.

\section{Violencia psicológica o emocional}

En esta categoría se observaron comportamientos como: poner apodos, amedrentar, amenazar, dejar en ridículo, burlarse, ignorar, desatender, regañar, remedar, demostrar fortaleza física, imponer y mandar (Sierra, sf). El comportamiento que se presenta con más frecuencia son los apodos, ya que la mayoría de los niños se maltratan de esta forma entre sí. Los apodos, por lo general, hacen referencia a distorsiones cómicas de los nombres, cambio de género (masculino-femenino) y a atributos físicos de la víctima, buscando ridiculizarla y ponerla en vergüenza ante los demás. Otro comportamiento son las palabras soeces o groserías. Por esta misma línea se puede hacer mención de las ofensas a otros niños. Las ofensas pueden abarcar comentarios con burlas, groserías o relativas a las familias de la víctima. El miembro de la familia que más es objeto de ofensas e insultos es la mamá, fuerte motivo por el cual se puede desencadenar una riña que implique agresión física. 
Los alumnos que fueron seleccionados para hacer parte de la categoría de víctimas presentan una diferencia particular con los alumnos que hacen parte de la categoría de agresores y es que, aunque las víctimas también pueden presentar este tipo de comportamientos ofensivos y poner apodos o agredir a otro, no lo hacen de una forma tan fuerte ni agresiva, ni con la misma intensidad como sí lo hacen los alumnos agresores.

\section{Conductas de acoso y amenaza entre esco- lares (victimización)}

En las relaciones entre niños se pueden presentar comportamientos que conllevan a unos procesos de hostigamiento y victimización que se basan en comportamientos violento, conductas que conllevan a colocar a uno o varios alumnos en el papel de víctima de violencia, ya sea física o psicológica (ver categorías 3 y 4). Otra forma de victimizar es por medio de la asignación de apodos, comportamiento empleado cuando se intenta burlarse de otro, cuando se quiere dejar en ridículo a alguien por ofender o simplemente por molestar. También se pueden observar comportamientos como el de imponer o mandar, llevados a cabo en su mayoría por los alumnos seleccionados en la categoría de "agresores", que producen miedo entre sus demás compañeros y así sacar provecho de ello. Es una de las estrategias más utilizadas por estos niños, debido a que asumen el rol de los fuertes del salón, y para los demás es mejor obedecerles puesto que así evitan ser lastimados o maltratados. Otra forma de victimizar hace referencia a actitudes intimidatorias destinadas a colocar a solamente un niño como víctima de violencia de tipo psicológico o emocional por parte de un grupo de alumnos. Este tipo de victimización conlleva a que el grupo de alumnos se reúna y determine una víctima específica, dejando a esta en una posición de la que difícilmente se puede librar, ya que sus agresores son mayoría. La victimización y las formas de amedrentar a otros niños, igualmente, se pueden llevar a cabo de forma individual. Este tipo de amedrentamiento se presenta con mayor frecuencia en los niños clasificados en la categoría de "agresores", ya que pueden usar su condición y el papel que les adjudican sus compañeros de ser los "más fuertes".

Otra característica de los comportamientos que se observan en esta categoría tiene que ver con las conductas de "hostigamiento" que se presentan, entendiendo por "hostigamiento" las conductas por medio de las cuales un niño molesta de una manera constante y por un pe- riodo bastante corto a otro niño, para buscar que este último se moleste o se irrite y posiblemente se inicie una pelea o discusión. Logra generar una posición en la cual la víctima de tales conductas se muestre irritable y su nivel de tolerancia se supere. Los hostigamientos que se observan con más frecuencia son: golpear de manera constante y suave a otro compañero, hacerle zancadilla, colocar apodos varias veces en un lapso corto, entre otros. Las conductas de acoso y amenaza entre escolares, en todas las ocasiones que se presentan, tienen la particularidad de que las asume un niño o una niña que en ese determinado momento se considera como más fuerte que su víctima, en la seguridad de que es poco probable que la víctima vaya a reaccionar de forma violenta y se defienda, ya que lo que más se busca con estas formas de victimización es la provocación de miedo en la víctima y recibir por parte de los demás respeto, gracias a la popularidad que adquieren quienes practican estas conductas de victimización, al demostrar con hechos o con insinuaciones su condición de niños fuertes y agresivos.

\section{Variables en la escuela}

En relación con los agresores se puede afirmar que son niños que se pueden relacionar fácilmente con cualquiera de sus compañeros de curso. Así mismo, son alumnos a quienes les es muy fácil integrarse en cualquier grupo; sin embargo, si no llegan a ser aceptados por algún grupo, logran integrarse "a la fuerza" haciendo uso de mecanismos de poder. Por tal razón, se observa que estos niños pueden permanecer con distintos grupos en la escuela. No obstante, por lo general la preferencia de estos niños en cuanto a sus amistades son los mismos niños que hacen parte de la categoría de agresores. En muy raras ocasiones se les observa jugando con los niños víctimas o con las niñas $y$, si lo hacen, es muy corto el tiempo de permanencia que logran mantener en tales actividades. En los juegos que comparten con las niñas son los líderes y coordinadores del juego. Con respecto a la niña que hace parte de esta categoría, se observa en ella que no tiene mucha preferencia por juegos violentos.

Con referencia a las víctimas, son niños a quienes les cuesta trabajo poder relacionarse con distintos grupos, y solamente se sienten a gusto y logran relacionarse tranquila y cómodamente con las niñas de su salón, compartiendo con ellas todo el tiempo de la jornada escolar en distintos tipos de actividades como juegos o trabajos en clase realizados en grupo. El grupo de 
amigos de los niños víctimas lo conforman solamente niñas. Con respecto a la relación que estos niños mantienen con sus maestros, se observa que son alumnos que en ocasiones también pueden manifestar rebeldía con normas o reglas que sus respectivos maestros les planteen, y se enojan bastante con estos últimos. Sus maestros también pueden regañarlos con frecuencia debido a sus conductas irritantes para con los demás. Sin embargo, no son niños que presenten graves problemas de indisciplina en el salón. Los maestros también pueden identificar fácilmente a estos niños como los más agredidos por otros en el salón, y porque son niños que "se la pasan dando quejas".

Con respecto a los sitios de la escuela donde con más frecuencia se practican las conductas de victimización entre escolares, estos pueden fluctuar entre los baños, el patio de recreo y el salón de clases. Se menciona que estos lugares son fluctuantes, pues no se presentan grandes diferencias entre ellos a la hora de los comportamientos violentos. Sin embargo, en los recreos (en el patio) sí es posible observar un mayor número de comportamientos violentos por parte de los niños, ya sean de tipo físico o psicológico. En los momentos en los que no se encuentra un maestro vigilándolos, hay mayor cantidad de conductas violentas. También se observan más comportamientos violentos, en la medida en que haya más niños presentes en el patio de recreo al mismo tiempo. Otro sitio en el cual se presenta mayor número de conductas violentas es el parque externo a la escuela, ya que allí no median tantas normas como dentro de la institución.

\section{Variables familiares}

Se observa que los niños agresores hacen parte de familias en las cuales se presentan ejercicios de poder y dominio mediante el castigo con violencia física y psicológica hacia los más débiles, como mamás, madrastras, hijos, y hermanos menores -en la relación entre hermanos-. Sin embargo, dicha situación es negada o disminuida su intensidad por parte de los propios niños. El padre de familia es quien más maltrata a los hijos. Sin embargo, cuando hay padrastros o madrastras presentes, estos no intervienen en los castigos ni correcciones hacia los niños de manera violenta. No se presentan ocasiones en familia que incluyan el diálogo para corregir a los niños.

Es poco el tiempo que comparten padres e hijos, debido a largas y continuas jornadas laborales. Algo carac- terístico del trato de estas familias para con sus hijos es el hecho de que los tratan como personas mayores a su edad y las exigencias son bastantes y severas.

Por otro lado, en cuanto al ambiente familiar de los niños víctimas, también se encontró que en estas familias se evidencian momentos de maltrato hacia los niños por parte de sus madres (conviven con padrastros). De acuerdo con ello, el maltrato más característico en estos niños es el de tipo psicológico, con regaños, gritos o insultos, y en pocas ocasiones se acude al maltrato físico. Sin embargo, por lo general predomina más el trato amable y cariñoso. No se observan en estas familias ejercicios de poder ni relaciones de dominio-sumisión, aunque los hermanos menores son maltratados por los mayores. A la hora de compartir el tiempo con sus hijos, en estas familias sí hay bastante tiempo que se comparte entre padres e hijos.

\section{Representación social de violencia en la escuela}

En primer lugar se observa que los niños tienen una idea clara de que los conflictos entre compañeros que llevan a comportamientos violentos pueden ser resueltos por otras vías como el diálogo y la conciliación. Las peleas tienen una connotación negativa y mencionan que "eso es malo". Sin embargo, en los niños agresores se observa cómo no llevan a la práctica los planteamientos sobre medios no violentos, contrario a como sí lo hacen los niños clasificados en la categoría de víctimas. Por otra parte, no se observa de forma clara lo que los niños agresores entienden por "débiles”, ya que ellos mismos pueden agredir a cualquier niño, sin importar si son más fuertes que ellos. A pesar de esto, sí se tiene en claro el fenómeno del "duro del salón", ya que se observa cómo ciertos niños son considerados por los demás como agresivos, fuertes o "los duros del salón”. Por tal razón, son tratados con respeto o incluso con cierto sentimiento de temor, en la medida en que tales niños pueden agredir a cualquiera en cualquier momento.

Entre los mismos niños agresores, se consideran como tales en la medida en que pueden halagarse al comentar cómo es que agreden a sus demás compañeros. De la misma manera, los otros compañeros pueden reforzarles esas ideas y crear un posicionamiento claro del papel que deben tomar como agresores, ya que a la hora de presentarlos a otra persona, lo hacen como los "duros 
del salón", "el que le pega a todos", "el que los defiende de los demás” o "el más peleón y el más fuerte”. Las profesoras titulares de curso también tienen muy en claro quiénes son los niños más "agresivos de sus respectivos salones”, y los niños que por lo general son más victimizados. De esta forma se observa cómo ciertos niños, por sus comportamientos particulares, pueden ser estigmatizados como agresivos o como víctimas.

Otro aspecto importante a resaltar en esta categoría es con respecto a los juegos, vistos como "juegos violentos" en la medida en que presentan conductas que implican violencia física. Sin embargo, tales juegos no son vistos por los niños como agresivos, violentos o maltratadores, ya que "simplemente se trata de un juego". En estos escenarios no hay agresores ni víctimas y todos los participantes pueden ser en distintos momentos tanto víctimas como agresores. Por último, con respecto a la violencia escolar vista desde los maestros, se observa que para ellos, aunque existen situaciones de violencia en su escuela, no la consideran como un problema grave, ya que para ellos es "algo normal dentro de las relaciones entre pares escolares”. Si algunos lo tienen en cuenta, minimizan la problemática refiriéndose a ella como "un problema que solo es de unos pocos".

\section{Discusión}

Los resultados derivados de la presente investigación permiten abordar varios temas de discusión. En primer lugar, se observó que los niños agresores son alumnos que presentan varios rasgos y características típicas de agresión a otros niños empleando la violencia tanto física como psicológica (ver resultados). Tales rasgos y características típicos de los niños agresores permitieron dar un giro a la comprensión de la problemática enfrentada por muchas instituciones educativas en la actualidad, y que se expresa en el fenómeno de la victimización y las conductas de acoso y amenaza entre escolares. Aquí entran en acción relaciones particulares entre sus protagonistas (los agresores y las víctimas), quienes presentan características mencionadas en la investigación realizada por Olweus (1998). En comparación con otras investigaciones, los resultados brindan la oportunidad de acceder a un mejor conocimiento de los rasgos típicos que suelen caracterizar a los protagonistas del fenómeno de la violencia escolar, con el fin de implementar nuevas estrategias de intervención para contrarrestar conductas de violencia en las escuelas.
De la misma manera se podría hablar de los niños clasificados en la categoría de víctimas, quienes también presentan características típicas. Sin embargo, en comparación con la misma investigación de Olweus, estos niños -en el presente estudio- no son clasificados en las subdivisiones planteadas como víctimas pasivas o sumisas y víctimas provocadoras, ya que nuestros datos arrojaron situaciones en las cuales estas víctimas presentan características de uno y otro subtipo y, en ocasiones, hay ciertos comportamientos que no presentan y que sí caracterizan a la víctima típica de Olweus.

Otro fenómeno digno de mencionar es el de "el duro del salón”, estudiado por Parra, González, Moritz, Blandón y Bustamante (1994), caracterizado por ser uno de los personajes más violentos del salón -por lo general niño-, que pasa a convertirse en un líder, que debe demostrar ante los demás que es fuerte para pelear, y presenta comportamientos autohalagadores por su condición de victimario de otros de su salón, así como también actos en los cuales puede mostrar su fuerza en confrontaciones con otros pares y buscarles pelea durante su participación en juegos que impliquen violencia física o cuando defiende a sus compañeros víctimas de maltrato por parte de otros niños.

Así como esta investigación halla coincidencias en la manera de caracterizar a los protagonistas de la violencia escolar (agresores y víctimas) con otras investigaciones preliminares -en especial la realizada por Olweus (1998)-, también ubica claras divergencias. Una de ellas es la siguiente: en el curso del presente estudio no se presenciaron consecuencias graves como deserción escolar, trastornos depresivos graves o, en el peor de los casos, el suicidio, en los niños clasificados en la categoría de "víctimas". Estos niños asisten con gusto a su escuela y tienen una gran motivación por sus actividades escolares. De acuerdo con los datos encontrados, se puede pensar que en la escuela en la cual se realizó la investigación, el problema de la violencia es palpable y cotidiano. Se pudo observar que allí se presentan situaciones de violencia física y psicológica o emocional casi con igual frecuencia.

Se observó que hay ciertos niños que golpean a los demás, en lo que Marland (1997) llama el "matoneo", y lo que Olweus (1998) plantea como el fenómeno de victimización o "bullying victim", el cual se da cuando alguien aprovecha su condición de ser más grande o más fuerte para agredir de alguna forma a otros más débiles; y se observa en la existencia de un grupo de 
alumnos a quienes les gusta amedrentar a los demás y golpearlos, con la plena seguridad de que los agredidos quedarán en una situación de impotencia, ya que los agresores pueden ser más fuertes y podrían tomar posteriores represalias contra las víctimas si se llegan a quejar o a desquitar. La víctima no tienen otra salida que la de someterse a lo que sus agresores le impongan. No obstante, en el presente estudio no se observó a ningún niño como objetivo único de agresiones, ya que la víctima de los niños agresores puede ser cualquiera, sin importar si son más fuertes o más grandes que ellos, o si son niños o niñas. Por lo tanto, no se presenta el fenómeno de la victimización con unas víctimas específicas. Sin embargo, sí se encontraron dos niños que son victimizados con mucha más frecuencia en relación con sus demás compañeros de clase.

De esta manera, se considera necesario recalcar que no es necesario golpear a otra persona para que exista agresión; el simple hecho de regañar fuertemente, amenazar, poner en ridículo a los alumnos en frente de sus compañeros, ignorarlos o desatenderlos implica que la persona víctima de estos actos se sienta mal y agredida, trayendo como consecuencia, en muchos casos, la baja de su autoestima o sentimientos de soledad o tristeza (Sierra, sf).

Se presenta un hecho de particular interés en la investigación, relacionado con los “juegos violentos". Lo específico de los "juegos violentos" es que en ellos no hay victimización ni se involucran los papeles de víctima y agresor. Se trata de juegos violentos practicados por los niños tales como el karate, tae-kondo, "blade-blade", la guerra de caballitos o el rambo, que son los preferidos de los agresores. Estos niños tienen un particular interés por este tipo de juegos que involucran violencia física, en los cuales participan con frecuencia y de manera muy activa. Las agresiones hacia los otros, los puños, patadas o empujones, no son vistos como actos de violencia, porque según su testimonio se trata de "un juego": "solamente estaban jugando". Tampoco se interpreta que los participantes de tales juegos sean víctimas o agresores, ya que el objetivo de estos últimos no es agredir, maltratar, violentar o causar daño físico al otro -aunque todo esto puede suceder-, sino divertirse. Si se trata de un juego, no hay por qué sentirse maltratado, simplemente juegan. En los juegos violentos todos pueden ser víctimas o agresores en cualquier etapa del mismo.

Por otro lado, es importante tener en cuenta el contexto en el cual conviven los niños alumnos de esta institución: un barrio en el cual se presentan altos niveles de violencia diariamente manifestada en atracos, violaciones, peleas y homicidios, que los niños presencian. Los niños agresores son quienes más presencian situaciones de violencia en sus hogares, ya sean las realizadas hacia los mismos niños, como castigos o maltratos físicos y psicológicos, o hacia otros miembros de la familia. En estos hogares se llevan a cabo ejercicios de poder y de sumisión y se presencian situaciones de violencia, lo cual lleva a pensar que los niños las reproducen en el colegio. Son personas que así mismo van a maltratar a otros niños en su escuela. Se podría interpretar la problemática de la violencia en las escuelas, y en especial la situación de los niños agresores, a partir de la teoría de Bandura y Walters (1963), en la perspectiva del aprendizaje social. Estos autores plantean que los niños pueden sufrir situaciones de violencia en sus hogares y reproducir comportamientos agresivos si se les presenta la ocasión oportuna para ello, ya que constantemente se encuentran rodeados de modelos agresivos. Son varios los modelos de violencia presenciados por niños de este sector de la ciudad, entre sus familias y en la comunidad del barrio donde viven.

Así mismo, al analizar dicho fenómeno de violencia en las escuelas, se puede observar cómo el hacinamiento podría ser considerado como una variable que influye en la generación de comportamientos violentos. De esta manera, se podría establecer que es importante diseñar y construir instituciones con espacios amplios en los cuales los niños tengan la oportunidad de jugar, correr y desplazarse con tranquilidad, sin el riesgo de tropezarse constantemente contra otro y crear, a partir de este incidente, una pelea. Se debe contar con espacios físicos en los cuales se pueda llevar a cabo un buen proceso de convivencia y aprendizaje en la escuela.

También se observó que cuando los niños son trasladados a otros sitios para su recreación, como el parque, se esperaba que los comportamientos violentos disminuyeran. Sin embargo, se observó que en otros contextos externos a la escuela también se presentan comportamientos violentos, incluso con mayor frecuencia e intensidad que dentro de la institución. De esta forma, surge otra posible interpretación con respecto a las conductas violentas entre pares, y es que estos niños consideran el parque como un contexto externo al de la escuela, en la que se percibe mayor vigilancia y presencia constante de maestros, hay unas normas cuya inobservancia conlleva a sanciones disciplinarias y está la oficina de la rectoría, tan temida por todos los niños. En cambio, es posible que los alumnos consideren sitios como el parque como el contexto de la calle, en el cual se desenvuelven cotidianamente, en 
el que las relaciones entre pares conllevan prácticas que no están mediadas por normas ni reglas institucionalizadas y se pueden ejercer más conductas de violencia física o psicológica, sin el temor a repercusiones como el castigo o la citación a sus respectivos padres. En este contexto de la calle se observa más agresividad que en la escuela. Varios niños que no son violentos en la escuela también participan en conductas que implican violencia, tanto de forma física como psicológica. Se podría concluir que dichos niños se encuentran en un ambiente promotor de la violencia y es así como la encuentran como algo rutinario para ellos.

De regreso al contexto escolar, es conveniente analizar cómo los maestros representan el problema de la violencia escolar como una variable que influye en el mantenimiento o eliminación de los comportamientos violentos entre pares. Durante el curso de la investigación fue registrada gran cantidad de comportamientos violentos de los niños de esta institución y, sin embargo, las maestras titulares de curso comentan que "en sus salones no se presentan conductas agresivas entre sus niños". A pesar de esto, ante la violencia y maltrato entre escolares se observa una acción directa por parte de los maestros, con su intervención oportuna y no permitiendo que pasen impunes en el contexto escolar. Intervienen para solucionar los conflictos entre alumnos con acciones como los llamados de atención, regaños, citaciones a los padres de familia, o mediante sanciones disciplinarias como castigos, no salir al recreo o enviar al agresor a la rectoría o a donde la orientadora. También existen algunos casos en que se presentan situaciones de violencia física y psicológica entre los alumnos y no hay ninguna intervención por parte de las maestras titulares de curso. Las maestras comentan, como ya se dijo, que "en sus respectivos cursos no se presentan conductas agresivas ni violentas" y que "eso es problema de unos pocos". En estos casos pareciera que la violencia escolar pasara desapercibida. Cuando los niños víctimas de algún tipo de maltrato plantean la queja ante su profesora y su información no es atendida, la víctima puede hacer justicia por su propia cuenta y ejercer un acto vengativo contra su agresor, de manera violenta. Esta situación por la que atraviesan muchas instituciones educativas está causada por la desatención de todos los problemas de violencia.

La violencia es un problema ante el que no debemos adoptar una posición pasiva y dejar que el tiempo pase sin intervenir adecuada y oportunamente; es un problema que está presente en nuestra realidad y más cerca de lo que creemos. Por violencia debemos dejar de pen- sar únicamente en los conflictos armados que aquejan a nuestro país, al igual que ponerle atención solamente a los casos de violencia física con consecuencias graves como la muerte. Tal como lo plantea Camargo (1997), al penetrar en el problema de la violencia escolar se presentan varias dificultades que crean una gran ambivalencia: la problemática de la violencia es trabajada muy escasamente, y esta es a su vez la razón por la cual no se reconoce su existencia ni se reflexiona sobre ella. Solamente se reflexiona en casos excepcionales.

Para llevar a cabo procesos de intervención y prevención del fenómeno de la victimización entre escolares y erradicar relaciones que tengan como protagonistas agresores y víctimas, es necesario realizar un trabajo conjunto entre familias y escuelas, ya que, como lo plantea Olweus (1998), a pesar de que la escuela es la institución que tiene la principal responsabilidad en la solución de los problemas que se presentan entre agresores y víctimas en su contexto, también es necesaria la intervención activa por parte de los padres de familia.

\section{BIBLIOGRAFÍA}

Alvarado, G. (1995). La Institución: Una categoría a reconstruir. Nómadas, 2: 31-38.

Angulo, B. (2003). Violencia escolar, un fenómeno mundial. Aula Urbana, 40 (abril-mayo): 20-21.

Ardila, A. (1999). La relación entre el adolescente pandillero y la escuela. En Vida de maestro. Violencia en la escuela. Bogotá: IDEP.

Armenta, T. (1999). Prevención de violencia y maltrato escolar. Bogotá: Pontificia Universidad Javeriana, Centro Universidad Abierta.

Bandura, A. y Walters, R. (1963). Patrones de reforzamiento y conducta social: agresión. En Megargee, E. y Hokanson, J. (1976). Dinámica de la agresión. México: Trillas Editorial.

Blanco, C., Docal, M., y Villamizar, M. (sf). Marginalidad y violencia. Bogotá: Instituto de Estudios Sociales Juan Pablo II. 
Camargo, M. (1997). Violencia escolar y violencia social. Revista Colombiana de Educación, 34: 5-24.

Campart, M. y Lindström, P. (1997). Intimidación y violencia en las escuelas suecas. Una reseña sobre investigación y política preventiva. Revista de educación. La violencia en los centros educativos, 313 (mayo-agosto).

Fernández, R. (sf). La violencia en los colegios. Una revisión bibliográfica. Consultada en http://averroes.cec. junta-andalucía.es/san_hermenegildo/violen.htm.2003

Freeman, D. (1977). La agresión humana en perspectiva antropológica. En Carthy, D. y Ebling, E. (1977). Historia natural de la agresión. México; Siglo XXI Editores.

Funk, W. (1997). Violencia escolar en Alemania. Estado del arte. Revista de educación. La violencia en los centros educativos, 313 (mayo-agosto).

Goetz, J. y LeCompte, M. (1988). Etnografía y diseño cualitativo en investigación educativa. Madrid: Morata.

Gómez, J. y Ramírez, P. (2000). La representación infantil del mundo social en el aula de clase: Las nociones sociales. Bogotá: Instituto de Estudios e Investigaciones Educativas.

Gunn, J. (1976). Violencia en la sociedad humana. Buenos Aires: Editorial Psique.

Instituto para la Investigación Educativa y el Desarrollo Pedagógico, IDEP. (1999). Vida de maestro. Violencia en la escuela. Bogotá: IDEP.

Jácome, C. (2003). Una mirada a la relación educadoralumno: a propósito de los límites de la educación. Curso psicoanálisis y educación. Sociedad Colombiana de Psicoanálisis, Bogotá.

Kogan, A. (1994). Resolución de conflictos: Un enfoque psicosociológico. México: Fondo de Cultura Económica.

Ledesma, A. (1980). El problema de la agresividad en el hombre contemporáneo. En Ledesma, A. (1980). I Curso monográfico sobre agresividad. Salamanca: Departamento de Psiquiatría Médica de la Universidad de Salamanca.
Marland, M. (1997). El matoneo en los colegios y el maltrato infantil. Revista de la Sociedad Colombiana de Psicoanálisis, 22 (2).

McCord, W., McCord, J. y Howard, A. (1961). Correlativos familiares de la agresión en niños no delincuentes. En Megargee, E. y Hokanson, J. (1976). Dinámica de la agresión. México. Trillas Editorial.

Mejía, S. (1997). Investigación sobre el maltrato infantil en Colombia (tomo I ). Colombia: Instituto Colombiano de Bienestar Familiar.

Ochoa, R. (2000). Violencia escolar. Fundamentos sociológicos. Consultado en http://roble.pnticmec.es./ fromero/fundamen.htm.2003

Olweus, D. (1998). Conductas de acoso y amenaza entre escolares. Madrid: Morata.

Ortega, R. (1997). El proyecto Sevilla anti-violencia escolar: Un modelo de intervención preventiva contra los malos tratos entre iguales. Revista de educación. La violencia en los centros educativos, 313 (mayo-agosto).

Ortega, R. y Mora-Merchán, J. (1997). Agresividad y violencia. El problema de la victimización entre escolares. En: Revista de educación. La violencia en los centros educativos, 313 (mayo-agosto).

Parodi, M. (1999). Las claves las tienen los niños. En Vida de maestro. Violencia en la escuela. Bogotá: IDEP.

Parra, R., González, A., Moritz, O., Blandón, A. y Bustamante, R. (1994). La escuela violenta. Colombia: Fundación FEs.

Pastor, G. (1980). La conducta agresiva desde el punto de vista de la psicología social. En Ledesma, A. (1980). I Curso monográfico sobre agresividad. Salamanca: Departamento de Psiquiatría Médica de la Universidad de Salamanca.

Peláez, S. (1991). La escuela como agente socializador y la violencia. En: Educación y cultura, 24: 22-31..

Rivarola, J. (1993). Reflexión sobre la violencia. Asunción: Arandura Editorial. 
Ruiz, J. (2002). Violencia armada en las escuelas: elementos para la perfilación. En Simposio nacional de Psiquiatría y Psicología Forense (memorias). Bogotá: Universidad de La Salle.

Sierra, C. (sf). Manifestaciones de violencia en la escuela. Seminario investigación cualitativa, Bogotá, Pontificia Universidad Javeriana, Facultad de Psicología.

Taylor, S. y Bogdam, R. (1996). Introducción a los métodos cualitativos de investigación. La búsqueda de significados. Barcelona: Paidós.

Valdés, M. (1991). La violencia ronda la escuela. Los niños no solo la padecen, la asumen. Educación y cultura, 24: $12-17$

Villa, M. y Moncada, R. (1998). Ciudad educadora en Colombia. Bogotá: Corporación Región.

Yorke, C. (1997). ¡Al caído, caerle! Un acercamiento psicoanalítico a los problemas del matoneo. Revista de la Sociedad Colombiana de Psicoanálisis, 2 (22). 